\title{
Lumbar microdiscectomy and lumbar decompression improve functional outcomes and depression scores
}

Authors Suzanne Tharin, Eric Mayer, Ajit Krishnaney

Institution Center for Spine Health, Cleveland Clinic Foundation, Cleveland, OH, USA

\section{ABSTRACT}

Study design: Retrospective review.

Introduction: Lumbar radiculopathy and claudicant leg pain are common degenerative spinal conditions often treated by elective microdiscectomy or decompression. Published outcome data for these procedures have focused on improvement in pain scores, and not on grounded functional outcome or depression scores [1-3]. Moreover, depression is considered by many surgeons to be a red flag for poor outcome for surgical treatment. We asked what effect lumbar microdiscectomy and laminectomy procedures had on functional outcome and depression scores in our clinical population.

Methods: Beginning in January 2010, the following outcome data were prospectively gathered before and after surgery from all patients at the Cleveland Clinic undergoing either lumbar microdiscectomy or lumbar decompression: EQ-5D (EuroQOL, quality-of-life measure), PHQ-9 (measure of depressive symptoms), PDQ (pain disability questionnaire), and Rankin scores (disability or dependence in daily activities).

Results: The mean EuroQOL scores improved by 35\% (from 0.4-0.75 of a maximum of 1.0) for both microdiscectomy and lumbar laminectomies. The mean PHQ-9 scores (measure of depressive symptoms) significantly improved for most patients undergoing either procedure. In line with previously published reports, we also found improvement in Rankin scores and Pain Disability Questionnaire scores.

Conclusions: Our outcome data indicate that microdiscectomy and lumbar decompression not only reduce disability and pain but also improve depressive symptoms and overall quality of life for patients. These findings support operative treatment of lumbar radiculopathy and neurogenic claudication including treatment performed in the depressed population. 


\section{REFERENCES}

1. Weinstein JN, Lurie JD, Tosteson TD, et al (2006) Surgical vs nonoperative treatment for lumbar disk herniation: the Spine Patient Outcomes Research Trial (SPORT) observational cohort. JAMA; 296(20):2451-2459.

2. Weinstein JN, Lurie JD, Tosteson TD, et al (2008) Surgical versus nonoperative treatment for lumbar disc herniation: four-year results for the Spine Patient Outcomes Research Trial (SPORT). Spine (Phila Pa 1976); 33:2789-2800.

3. Weinstein JN, Tosteson TD, Lurie JD, et al (2006) Surgical vs nonoperative treatment for lumbar disk herniation: the Spine Patient Outcomes Research Trial (SPORT): a randomized trial. JAMA; 296(20):2441-2450. 